\title{
Painless Cervical Lymphadenopathy in an Elderly Patient - A Rare Case of Rosai-Dorfman Disease and Hodgkin's Lymphoma
}

\author{
Shi Ying Tan, $M B, B C h, B A O^{*}$, Pemasiri Upali Telisinghe, MBBS and Justin Fook Siong \\ Keasberry, MB ChB, FRACP
}

Raja Isteri Pengiran Anak Saleha Hospital, Brunei Darussalam

*Corresponding author: Tan Shi Ying, Internal Medicine, Raja Isteri Pengiran Anak Saleha Hospital, Jalan Putera AlMuhtadee Billah, Bandar Seri Begawan BA 1712, Brunei Darussalam, Tel: +673-8365155

\begin{abstract}
Rosai-Dorfman disease is a rare, benign disease presenting with extensive lymphadenopathy. In this case, an 81-yearold gentleman with previous pulmonary tuberculosis, presented with painless cervical lymphadenopathy associated with generalized weakness, loss of appetite, and cough. The causes of cervical lymphadenopathy in elderly are comprehensive; hence thorough history taking, examination, and appropriate investigations are vital in diagnosing diseases. If blood investigations and imaging are inconclusive, a lymph node biopsy is recommended. $\mathrm{He}$ was later diagnosed with Rosai-Dorfman disease associated with Hodgkin's lymphoma and dexamethasone treatment was initiated. Concomitant diagnoses are rare, but should not disregarded. Regular monitoring is required as immune dysregulation may exacerbate quiescent, treated pulmonary tuberculosis.
\end{abstract}

\section{Keywords}

Lymphadenopathy, Rosai-Dorfman disease, Histiocytosis, Sinus

\section{Introduction}

Rosai-Dorfman Disease (RDD) is an uncommon disorder first described by Destombes in 1965. Rosai and Dorfman subsequently published more data of RDD in 1972 [1]. The condition presents commonly in males in the first and second decades, although any age could be affected. RDD is also known as sinus histiocytosis with massive lymphadenopathy (SHML), characterized by benign proliferation of histiocytes within lymph node sinuses and lymphatics in extra nodal sites. We present a unique case of cervical lymphadenopathy in elderly, later diagnosed as RDD associated with Hodgkin's lymphoma below.

\section{Case Description}

Mr. N, an 81-year-old gentleman, with a background history of dementia, seizures, and previously treated pulmonary tuberculosis, presented to the hospital with a week history of generalized body weakness and loss of appetite. He denied any fever, but complained of cough with white sputum. His pre-admission medications include levetiracetam $500 \mathrm{mg} \mathrm{BD}$, rivastigmine 1.5 $\mathrm{mg} \mathrm{BD}$, and amlodipine $5 \mathrm{mg} \mathrm{OD}$. On examination, he had palpable pea sized, painless, enlarged left submandibular lymph nodes and inguinal lymph nodes bilaterally. His abdomen was soft, and non-tender but hepatomegaly was present. Other examination findings were unremarkable.

Significant laboratory results included a raised CRP of $5.0 \mathrm{mg} / \mathrm{dL}$, and raised ESR of $113 \mathrm{~mm} / \mathrm{hr}$. Bicytopenia with WCC 3.6, and $\mathrm{Hb}$ of 10.3. Total protein was raised at $122 \mathrm{~g} / \mathrm{L}$. Sodium was $123 \mathrm{mmol} / \mathrm{L}$; other UECs and LFTs were unremarkable. An ultrasound scan of the neck showed multiple enlarged lymph nodes in bilateral submandibular, anterior and posterior cervical triangles. CT thorax, abdomen and pelvis was done and showed multiple nodes measuring $5.7 \mathrm{~mm}$ to $9.2 \mathrm{~mm}$ in right para tracheal and sub carinal regions, $7.0 \mathrm{~mm}$ to 


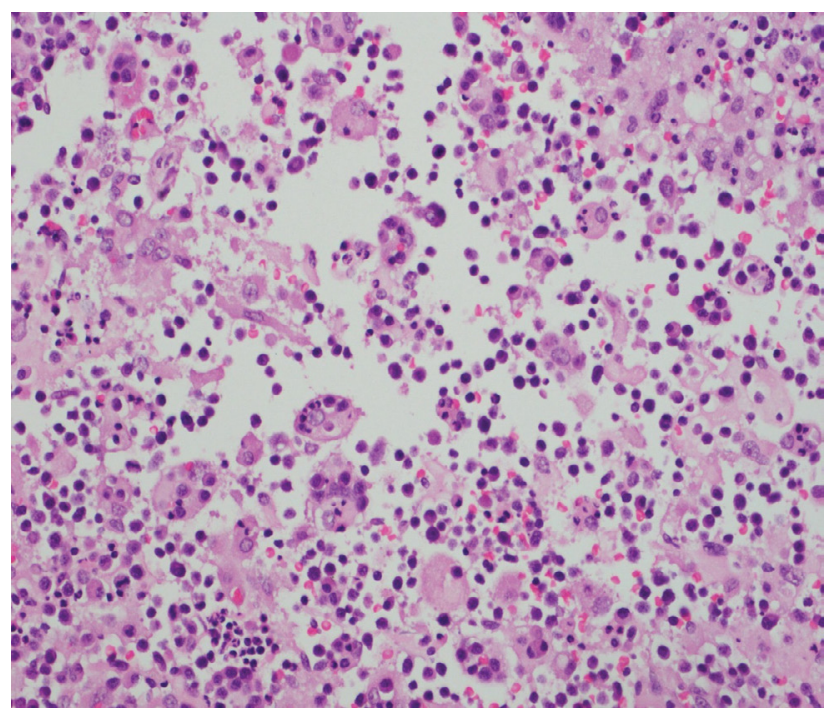

Figure 1: Dilated sinus with histiocytes showing emperipolesis.

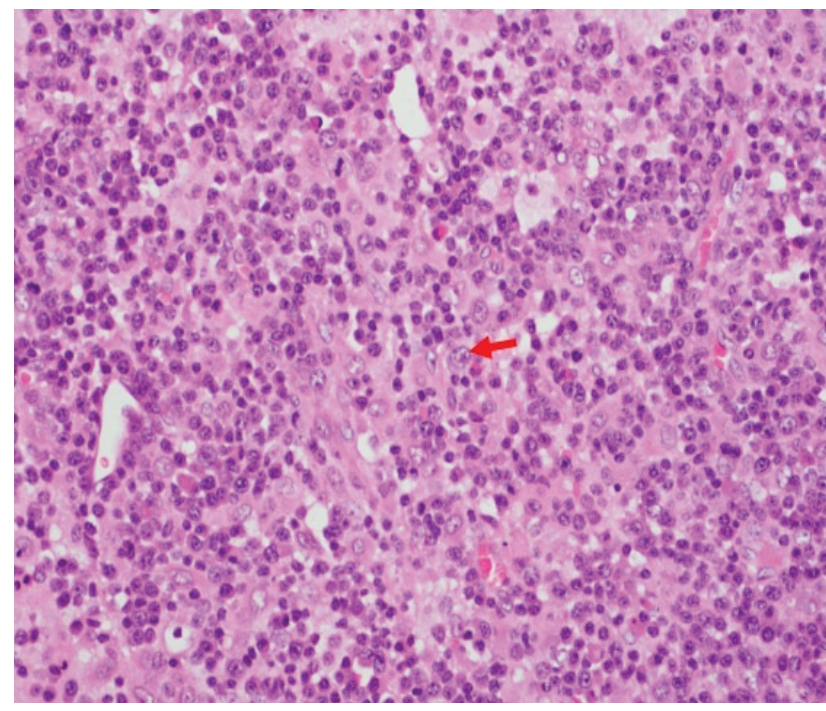

Figure 2: S-R like cells (arrow) noted in biopsy.

$28.4 \mathrm{~mm}$ in both axillae, abdominopelvic adenopathies noted at left paraaortic (largest measuring $2.1 \times 1.5 \mathrm{~cm}$ ), interaortocaval $(1.4 \times 1 \mathrm{~cm})$, retrocaval $(1.7 \times 1 \mathrm{~cm})$, portal $(2.6 \times 2.1 \mathrm{~cm})$, coeliac $(2.7 \times 1.9 \mathrm{~cm})$, mesenteric $(3.2 \times 1.8 \mathrm{~cm})$, bilateral external iliac (largest $2.3 \times 1.5$ $\mathrm{cm}$ on right $\& 1.5 \times 1.4 \mathrm{~cm}$ on left), bilateral inguinal (largest $2.3 \times 1.4 \mathrm{~cm}$ on right $\& 2.2 \times 1.4 \mathrm{~cm}$ on left).

Further work-up for possible diagnoses and immunocompromised status were done, which include Human Immunodeficiency Virus (HIV) serology, Hepatitis $B$ and $C$ serologies, triple tuberculosis acid fast bacteria (AFB) smears, antinuclear antibodies (ANA) and antidouble stranded DNA (anti-dsDNA) for autoimmune screening, and $\mathrm{HbA} 1 \mathrm{c}$ for diabetes mellitus; which were all negative. As his total protein was raised with anemia, work-up for multiple myeloma was done; lactate dehydrogenase was mildly raised at $267 \mathrm{U} / \mathrm{L}$, but urine Bence-Jones protein and serum paraprotein at electrophoresis were both not detected. Excision biopsy

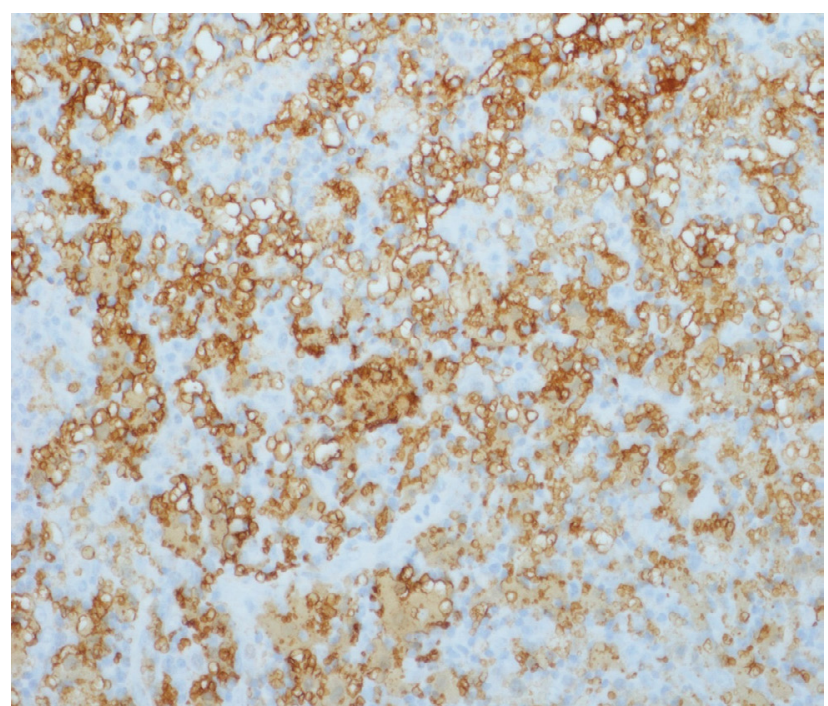

Figure 3: Histiocytes showing S100 positive immunostaining.

of the right neck node was then performed and specimen was sent for frozen section, showing dilated sinuses filled with histiocytes and lymphocytes. Histiocytes show emperipolesis (Figure 1). The surrounding tissue shows nodular arrangement with lymphocytic, plasma cell and Reed-Sternberg (R-S) like cell infiltrate along with a few eosinophils (Figure 2). The histiocytes within the sinuses show positive reaction with S-100 (Figure 3 ), while the R-S like cells show positive reaction with CD30 and CD15. Overall picture was RDD, associated with Hodgkin's lymphoma. As Mr. N is not a good candidate for chemotherapy, oral dexamethasone $4 \mathrm{mg}$ BD was initiated, along with allopurinol for risk of tumor lysis syndrome post steroids therapy.

Mr. N was re-admitted to the hospital 3 weeks later as his sputum cultures grew Mycobacterium tuberculosis complex. Two months intensive therapy of Isoniazid, rifampicin, ethambutol and pyridoxine were initiated, followed by seven months of isoniazid and rifampicin. His dexamethasone was subsequently reduced to $2 \mathrm{mg}$ BD.

\section{Discussion}

Patients with RDD usually present with nonspecific findings such as low-grade fever, cervical lymphadenopathy, normochromic anemia, elevated ESR, leukocytosis (mainly neutrophilia), and hyperglobulinemia [1]. Thesewereconsistentwith Mr. N's initial presentation, although he did not report any fever, and had leukopenia instead. Cervical lymphadenopathy in elderly is a common presenting complain, but could be caused by a vast array of diseases, including bacterial infections e.g. cat scratch disease; viral infections e.g. Hepatitis B, HIV; Mycobacterium tuberculosis; cancers e.g. leukemia and lymphoma, lymphoproliferative disorders e.g. Rosai-Dorfman disease, hemophagocytic lymphohistiocytosis; autoimmune disorders e.g. systemic lupus erythematosus, rheumatoid arthritis, dermatomyositis, or medications e.g. atenolol, captopril, 
carbamazepine, lamotrigine, and phenytoin [2]. Most patients with RDD present with chronic bilateral, painless cervical lymphadenopathy. Notably, if only one lymph node group was enlarged, this was commonly the submandibular region [1,3], which was similar to Mr. N's case. Mr. N also had inguinal and axillary lymph nodes; these were usually less prominent compared to cervical involvement in RDD.

Evaluation of lymphadenopathy causes should be based on history and physical findings, followed by routine blood investigations, HIV testing, and chest $X$-ray. If unremarkable, further assessments such as tuberculosis, ANA, syphilis, and heterophile test should be considered [4]. Mr. $\mathrm{N}$ has most of the investigations done, except for syphilis and heterophile test. None of his medications were identified to cause lymphadenopathy as well. For indefinite diagnosis, a lymph node biopsy is usually recommended.

In SMHL, histological findings of lymph node include capsular and pericapsular fibrosis, with numerous histiocytes containing lymphocytes in their cytoplasm (emperipolesis). Emperipolesis, the presence of an intact cell within cytoplasm of another cell, is of great diagnostic significance for RDD, despite not pathognomonic. In the initial phase of disease, prominent dilatation of sinuses was reported. Both emperipolesis and dilated sinuses were seen in our case too. These sinuses would later be occupied by mainly histiocytes, and other cells such as lymphocytes, plasma cells and occasional neutrophils, resulting in effacement of nodal structure $[1,3,5]$. Histiocytes characteristically demonstrate reactivity to CD11C, CD14, CD33, CD68 antigens and $\mathrm{S} 100$ protein. They are usually CD1 negative. Differential diagnoses for SHML include reactive sinus histiocytosis, Langerhans cell histiocytosis, malignant histiocytosis, hemophagocytic syndrome, tuberculosis, and lymphoma. Each of the differentials has characteristics to distinguish them from SHML. For instance, reactive sinus histiocytosis expresses CD68, but lack S100 and CD1a. On the contrary, Langerhans cells are reactive to $\mathrm{S} 100$ and CD1a. They also show Birbeck's granules on electron microscopy. Malignant histiocytosis demonstrates prominent cellular atypia and mitosis, which is rare in SHML. Hemophagocytic syndrome is associated with pancytopenia and hepatosplenomegaly; which are generally not present in SHML. SHML can be differentiated from tuberculous lymphadenitis as tuberculosis would show epithelioid cell granuloma with or without caseous necrosis. Lastly, Non-Hodgkin's lymphoma consist of lymphoid cells that show positivity for CD3 (T-cell), CD20 (B-cell), or bcl-2 (anti-apoptic) marker, while Hodgkin lymphoma contain RS cells reactive to CD15, CD30 or CD20; these are usually negative in SHML [3,5].

Mr. N's biopsy showed positive reaction to S-100, CD30 and CD15, which concluded he has a dual pathology of RDD with Hodgkin's lymphoma. A literature review done by Edelman, et al. showed 25 cases of RDD associated with Hodgkin and non-Hodgkin lymphoma, in which $70 \%$ of the dual pathologies were diagnosed simultaneously [6]. Sporadic RDD is the most common form of RDD, which include classic nodal, extranodal, neoplastic related, and immune disease related RDD. Classic RDD is the most prevalent type, which affects children and young adults in the first two decades. Extranodal disease is more common in older patients, which involve skin, nasal cavity, bone, orbital tissue, and central nervous system. As RDD can co-exist with neoplasia, pathological findings of RDD should be present in more than $10 \%$ of the tissue to establish neoplasia-associated RDD [7].

In addition, Mr. $\mathrm{N}$ was also later diagnosed with tuberculosis through sputum cultures and received treatment. His tuberculosis is likely a reactivation of disease as he was treated for it many years back. The Hodgkin's lymphoma and dexamethasone may suppress the cell-mediated response, which facilitated the mycobacterial infection $[8,9]$. There has also been documented literature on tuberculosis preceding onset of RDD with no known causative link; however, this could be attributed to high prevalence rate in Asian countries [10]. Several reports recorded patients present with symptoms mimicking tuberculosis, but were later diagnosed as RDD [11-13]. A study done in Pakistan also showed that the most common causes for lymphadenopathy include tuberculosis (70.45\%), followed by reactive lymphadenitis (13.63\%), metastases (11.36\%), lymphoma (4.54\%), and chronic nonspecific lymphadenitis (2.27\%) [4]. There is potential for misdiagnosis as RDD, tuberculosis and Hodgkin's lymphoma as they present with similar signs and symptoms such as fever, cough, loss of appetite, and adenopathy [8]. Therefore, it is important to properly diagnose patients to ensure they receive the right treatment.

Lymphadenopathy in RDD will eventually regresses without treatment, despite waxing and waning over months to years. Treatment with corticosteroid usually shows good clinical response. Other treatments include alkylating agents, vinca alkaloids, and low dose interferon. Radiation therapy and surgical treatment can be used for life threatening obstructions due to pressure exerted by lymph nodes. Patients generally have good prognosis, unless they have associated immunological pathologies, extra nodal involvement especially of kidney and liver, or at a younger age group $[3,5]$. Long-term follow up is essential to monitor course of disease, and reactivation of tuberculosis.

\section{Conclusion}

RDD is usually diagnosed clinically, and confirmed by laboratory investigations. Albeit rare, it should remain as a differential diagnosis for cervical lymphadenopathy. 
Patients may present with concomitant pathologies; hence, it is important to do a full work-up for patients to ensure proper treatments can be delivered. Tuberculosis reactivation should always be taken into consideration, especially in highly endemic countries.

\section{Funding}

Nil.

\section{Conflicts of Interest}

The authors claim no conflicts of interest.

All authors contribute equally in this manuscript.

\section{References}

1. Rosai J, Dorfman RF (1972) Sinus histiocytosis with massive lymphadenopathy: A pseudolymphomatous benign disorder. Analysis of 34 cases. Cancer 30: 11741188.

2. Ferrer RL (2021) Evaluation of peripheral lymphadenopathy in adults.

3. Kumar B, Karki S, Paudyal P (2008) Diagnosis of sinus histiocytosis with massive lymphadenopathy (RosaiDorfman disease) by fine needle aspiration cytology. Diagn Cytopathol 36: 691-695.

4. Iqbal M, Subhan A, Aslam A (2010) Frequency of tuberculosis in cervical lymphadenopathy. J Surg Pak 15: 107-109.
5. Sharma S, Bhardwaj S, Hans D (2010) Rosai-Dorfman disease. JK Science 12: 194-196.

6. Edelman A, Patterson B, Donovan K, Malone J, Callen J (2019) Rosai-Dorfman disease with a concurrent mantle cell lymphoma. JAAD case reports 5: 40-43.

7. Bruce-Brand C, Schneider JW, Schubert $P$ (2020) RosaiDorfman disease: An overview. J Clin Pathol 73: 697-705.

8. Reddy RC, Mathew M, Parameswaran A, Narasimhan R (2014) A case of concomitant hodgkin's lymphoma with tuberculosis. Lung India 31: 59-62.

9. Centkowski P, Sawczuk-Chabin J, Prochorec M, Warzocha K (2005) Hodgkin's lymphoma and tuberculosis coexistence in cervical lymph nodes. Leuk Lymphoma 46: 471-475.

10. Hsiao C-H, Tsai T-F, Yang T-H, Liu C-M (2006) Clinicopathologic characteristics of rosai-dorfman disease in a medical center in northern taiwan. J Formos Med Assoc 105: 701-707.

11. Zacharia B, Manicam K (2020) Primary rosai-dorfman disease of the proximal radius masquerading as tuberculosis of elbow. Cureus 12: e7858.

12. Parkash O, Yousaf MS, Fareed G (2019) Rosai-Dorfman's disease, an uncommon cause of common clinical presentation. J Pak Med Assoc 69: 1213-1215.

13. Elo-Ilo JC, Odinaka KK, Ukah CO (2016) Rosai-Dorfman disease in a 12-year-old nigerian male. S Afr J Child Health 10: $237-238$. 